\title{
A genetic study based on PCNA-ubiquitin fusions reveals no requirement for PCNA polyubiquitylation in DNA damage tolerance
}

\author{
Judit Z. Gervai ${ }^{\mathrm{a}}$, Judit Gálicza ${ }^{\mathrm{b}}$, Zoltán Szeltner ${ }^{\mathrm{a}}$, Judit Zámborszky ${ }^{\mathrm{a}}$, Dávid Szüts ${ }^{\mathrm{a}, *}$ \\ a Institute of Enzymology, Research Centre for Natural Sciences, Hungarian Academy of Sciences, Magyar tudósok krt. 2, Budapest, H-1117, Hungary \\ b Macromolecular Crystallography Group, Max Planck Institute for Biophysical Chemistry, Am Fassberg 11, Göttingen, 37077, Germany
}

\section{A R T I C L E I N F O}

\section{Keywords:}

DNA damage bypass

Translesion synthesis

Template switching

PCNA

PCNA monoubiquitylation

PCNA polyubiquitylation

\begin{abstract}
A B S T R A C T
Post-translational modifications of Proliferating Cell Nuclear Antigen (PCNA) play a key role in regulating the bypass of DNA lesions during DNA replication. PCNA can be monoubiquitylated at lysine 164 by the RAD6RAD18 ubiquitin ligase complex. Through this modification, PCNA can interact with low fidelity Y family DNA polymerases to promote translesion synthesis. Monoubiquitylated PCNA can be polyubiquitylated on lysine 63 of ubiquitin by a further ubiquitin-conjugating complex. This modification promotes a template switching bypass process in yeast, while its role in higher eukaryotes is less clear.

We investigated the function of PCNA ubiquitylation using a PCNA ${ }^{K 164 R}$ mutant DT40 chicken B lymphoblastoma cell line, which is hypersensitive to DNA damaging agents such as methyl methanesulfonate (MMS), cisplatin or ultraviolet radiation (UV) due to the loss of PCNA modifications. In the PCNA ${ }^{K 164 R}$ mutant we also detected cell cycle arrest following UV treatment, a reduced rate of damage bypass through translesion DNA synthesis on synthetic UV photoproducts, and an increased rate of genomic mutagenesis following MMS treatment. PCNA-ubiquitin fusion proteins have been reported to mimic endogenous PCNA ubiquitylation. We found that the stable expression of a PCNA ${ }^{\mathrm{K} 164 \mathrm{R}}$-ubiquitin fusion protein fully or partially rescued the observed defects of the $P C N A^{K 164 R}$ mutant. The expression of a PCNA ${ }^{\mathrm{K} 164 \mathrm{R}}$-ubiquitin ${ }^{\mathrm{K} 63 \mathrm{R}}$ fusion protein, on which the formation of lysine 63-linked polyubiquitin chains is not possible, similarly rescued the cell cycle arrest, DNA damage sensitivity, reduction of translesion synthesis and increase of MMS-induced genomic mutagenesis. Template switching bypass was not affected by the genetic elimination of PCNA polyubiquitylation, but it was reduced in the absence of the recombination proteins BRCA1 or XRCC3. Our study found no requirement for PCNA polyubiquitylation to protect cells from replication-stalling DNA damage.
\end{abstract}

\section{Introduction}

The successful bypass of sites of DNA damage during the replication of chromosomal DNA is essential for cell survival and the maintenance of genome integrity. PCNA is a key protein in DNA replication, DNA repair and cell cycle regulation [1,2]. Post-translational modifications (ubiquitylation or sumoylation) of PCNA play an important role in regulating its function in lesion bypass during DNA replication. The conserved lysine 164 side chain can be monoubiquitylated by the RAD6-RAD18 ubiquitin ligase complex in a variety of organisms from yeast to higher eukaryotes [3-5]. Monoubiquitylated PCNA recruits low fidelity Y family DNA polymerases ( $\mathrm{Pol} \eta$, Pol $\kappa$, Pol $\imath$ ) to promote TLS [6-8]. Monoubiquitylated PCNA can also undergo further ubiquitylation catalysed by Ubc13-Mms2 and the Rad5 ubiquitin ligase in yeast, or UBC13-MMS2 and the Rad5 orthologues HLTF and SHPRH in human cells, resulting in the build-up of short lysine 63 (K63)-linked polyubiquitin chains $[9,4,10,11]$. This modification is different from the K48-linked polyubiquitin chain that serves as a degradation signal. The K63-linked polyubiquitylation of PCNA was genetically tied to a Rad18and Rad5-dependent error-free bypass mechanism in yeast that uses the sister chromatid as an alternative template to extend the stalled DNA strand past a lesion in the template [12]. Two alternative mechanisms have been proposed for such a template switching process. Twodimensional gel analysis of replication intermediates demonstrated a process that operates through a recombinational mechanism, forming sister chromatid junctions behind the replication fork [13]. Indeed, PCNA polyubiquitlylation-dependent template switching in yeast is dependent on the key HR protein Rad51, demonstrating a functional

Abbreviations: PCNA, proliferating cell nuclear antigen; Ub, ubiquitin; TLS, translesion synthesis; TSw, template switching; MMS, methyl methanesulfonate; UV, ultraviolet radiation; $\mathrm{SNV}$, single nucleotide variation; HR, homologous recombination

* Corresponding author.

E-mail address: szuts.david@ttk.mta.hu (D. Szüts). 
overlap between template switching and HR [13,14]. An alternative mechanism for template switching through the reversal and regression of stalled replication forks $[15,16]$ is supported by the structure-specific helicase ability of Rad5 [17], though recently the helicase activity of Rad5 was shown to be dispensable for template switching [18].

Lesion bypass through the use of an alternative template has also been demonstrated in higher eukaryotes [19,20], and increased UVinduced mutagenesis upon overexpressing K63R mutant ubiquitin indirectly implicates PCNA polyubiquitylation in the process [21]. However, direct evidence for the connection between PCNA polyubiquitylation and damage bypass is lacking. SHPRH promotes mutagenic bypass on UV lesions, while HLTF also regulates PCNA monoubiquitylation [22], which likely contributes to its effect in DNA damage tolerance.

We sought to establish a clear genetic assay for the effect of PCNA polyubiquitylation in DNA damage tolerance. As it is not clear which enzymes are capable of polyubiquitylation in higher eukaryotes $[23,24]$, we opted for the use of PCNA-ubiquitin (PCNA-Ub) fusion proteins in which the ligation of ubiquitin units can be controlled by altering the target lysines. The $\mathrm{N}$ - or C-terminal linear fusion of one ubiquitin molecule to PCNA has been shown to partially substitute for the endogenous ubiquitylation of PCNA in damage tolerance in $S$. cerevisiae [25,26], S. pombe [27] and cultured human cells [28]. The fusion proteins in these studies allowed the potential formation of polyubiquitin chains on the fused ubiquitin moiety. In our study we used combinations of mutations of the target lysines in the endogenous PCNA gene and in expressed C-terminal PCNA-Ub fusion proteins to genetically allow or inhibit PCNA polyubiquitylation. Our results suggest that PCNA polyubiquitylation is not required for DNA damage tolerance or the prevention of mutagenesis in chicken DT40 cells.

\section{Materials and methods}

\subsection{Transgenic expression constructs}

For the non-fusion PCNA expression constructs, the full length chicken PCNA coding sequence was amplified from the cDNA of the wild type DT40 cell line using primers CAGCTAGC(NheI)CAGTCGAC(SalI)ATGTTCGAGGCGCGGCTTGTG (primer P1) and CAGGATCC(BamHI)TTAAGAGCCTTCTTGCTGGTCTTCAATC, and cloned into the multiple cloning site of pIRES2-EGFP with SalI and BamHI. PCNA-IRES-EGFP was amplified with primers P1 and CAGCGGCCGC(NotI)TTACTTGTACAGCTCGTCCATGC, cloned into the pExpress-derived pXpsn2 $[29,30]$ using SalI and NotI, cut with SpeI and cloned into NheI-cut pLoxBsr or pLoxPuro [29]. For the PCNA-Ub fusion expression constructs, the PCNA coding sequence was amplified using primers P1 and CACAATTG(MfeI)CTCGGCAGTGCCAGAGCCTTCTTGCTGGTCTTCAATCT, thereby removing the stop codon and inserting a C-terminal GTAEQL peptide linker in analogy with the GTAEIQ linker used in $S$. cerevisiae [25]. A ubiquitin coding sequence without the C-terminal two glycines was also amplified from cDNA using primers GTCACAATTG(MfeI)ATGCAGATCTTCGTGAAAACGTTGACTGG and GTCAGGATCC(BamHI)CTA(stop)CCTCAGGCGCAGCACCAGATG. The PCNA and ubiquitin sequences were assembled in a vector with a purpose-made multiple cloning site. The PCNA K164R modification and the ubiquitin K63R modification were generated by site directed mutagenesis. The PCNA-Ub fusion sequences were subcloned using MluI into modified, non-loxable pLoxBsr/Puro plasmids, which were prepared by amplifying the IRES-EGFP sequence from pIRES2EGFP using primers CAGTCGAC(SalI)AGCAATTG(MfeI)TCACGCGT(MluI)CGCCCCTCTCCCTCCCC and CAGCGGCCGC(NotI)TTACTTGTACAGCTCGTCCATGCC, cloning into pXpsn2 using SalI and NotI, excising the transgene with SpeI and cloning into SpeI-cut pLoxBsr/Puro.

\subsection{In vitro ubiquitylation assay}

Human UBA1 was purchased from Sigma Aldrich. The complex of UBC13-MMS2 was expressed in baculovirus expression system. Plasmids containg the human UBC13 and MMS2 cDNA were purchased from Addgene and recloned into a modified pFL baculovirus vector [31]. The expression of MMS2 was under control of the p10 promoter, while the expression of UBC13 in frame with and $\mathrm{N}$-terminal $\mathrm{His}_{6}$-tag was controlled under the polh promoter of pFL. The complex was expressed in Sf9 insect cells and purified via the N-terminal histidine tag of UBC13 on a Ni-NTA (GE Healthcare) affinity column, followed by Q-Sepharose Hi-Trap $1 \mathrm{~mL}$ (GE Healthcare) and Superdex 200 Increase columns pre-equilibrated with $20 \mathrm{mM}$ Na-HEPES pH 7.5, $100 \mathrm{mM} \mathrm{NaCl}$, $5 \%$ glycerol. Human ubiquitin was amplified from human cDNA library and cloned into the pETM-11 vector. The protein was expressed in the Rosetta II E.coli strain and affinity purified on a $1 \mathrm{~mL}$ Ni-NTA (GE Healthcare) column, then further purified on Superdex 75 16/60 size exclusion chromatography column (GE Healthcare) pre-equilibrated with $20 \mathrm{mM}$ Na-HEPES pH 7.5, $100 \mathrm{mM} \mathrm{NaCl}, 5 \%$ glycerol. The full length human HLTF cDNA [10] was cloned into pFL under the polh promoter. The protein was expressed in Sf9 insect cells and was affinity purified in one step on amylose resin through its maltose binding protein (MBP) tag in buffer containing $40 \mathrm{mM}$ Na-HEPES pH 7.5, 10\% glycerol, $2 \mathrm{mM}$ 2-mercaptoethanol and $100 \mathrm{mM} \mathrm{NaCl}$. PCNA constructs were subcloned into the pET-15b vector (Novagen) and expressed in $E$. coli. The $\mathrm{N}$-terminal his-tagged fusion proteins were affinity purified on Ni-NTA Superflow resin (QIAGEN), followed by gel filtration on a Superose 12 column (GE Healthcare). Ubiquitylation reactions all contained $40 \mathrm{mM}$ Na-HEPES pH 7.5, $8 \mathrm{mM} \mathrm{MgCl}_{2}, 50 \mathrm{mM} \mathrm{NaCl}$, $1 \mathrm{mM}$ ATP, $250 \mathrm{nM}$ ubiquitin and $100 \mathrm{nM}$ PCNA substrate, plus $100 \mathrm{nM}$ UBA1, $250 \mathrm{nM}$ UBC13-MMS2 complex and $250 \mathrm{nM}$ HLTF as indicated. Reactions were incubated at $30{ }^{\circ} \mathrm{C}$ for $60 \mathrm{~min}$ and stopped with the addition of SDS sample buffer.

\subsection{Cell culture and colony survival assay}

This study used a wild type DT40 cell line [32], a $P C N A^{K 164 R / K 164 R}$ line referred to in the text as $P C N A^{K 164 R}$ [33], $X P A^{-}$and $X P A^{-}$ $P C N A^{K 164 R / K 164 R}$ cells [20]. XPA ${ }^{-} B R C A 1^{-/-}$and $X P A^{-} X R C C 3^{-/-}$ cell lines were generated by disrupting the single-copy $X P A$ gene in the respective heterozygous HR mutants, then disrupting the second allele of BRCA1 [34] and XRCC3 [35].

Cells were grown at $37{ }^{\circ} \mathrm{C}$ under $5 \% \mathrm{CO}_{2}$ in RPMI- 1640 medium supplemented with $7 \%$ fetal bovine serum, $3 \%$ chicken serum, $50 \mu \mathrm{M} 2$ mercaptoethanol and penicillin/streptomycin.

MMS, cisplatin and UV sensitivities were measured using colony survival assays; treated cells were plated in culture medium containing $1 \%$ methylcellulose (Sigma) using a tenfold dilution series, and surviving colonies were counted 10 days later.

\subsection{Shuttle plasmid lesion bypass assay}

600,000 cells were transfected with $0.1 \mu \mathrm{g}$ pQ-CPDs shuttle plasmid containing two synthetic T-T cyclobutyl pyrimidine dimers in a staggered arrangement [36]. The transfections were performed using a Nucleofector device (Lonza) as described previously [36]. The transfected cells were cultured at $37^{\circ} \mathrm{C}$ for $24 \mathrm{~h}$, then the plasmid was extracted using the Hirt protocol and digested with $10 \mathrm{U}$ DpnI restriction endonuclease in $10 \mu \mathrm{L}$ at $37^{\circ} \mathrm{C}$ for $30 \mathrm{~min}$ to destroy unreplicated dam methylated plasmids. The efficiency of plasmid replication was quantified from $\mathrm{qPCR}$ measurements as the amount of a recovered DpnI-susceptible plasmid fragment divided by the amount of a control fragment [36]. The digested DNA was purified and used as template for PCR amplification with Pfu Turbo (Agilent) of a $1369 \mathrm{bp}$ section of DpnI resistant replicated pQ-CPDs using primers CAGTCGACTTTTGATTTAGAATTGTCCAC and CAGCGGCCGCTTTGCAAGCAGCA- 
GATTACG. To determine in bulk the proportion of each common sequence outcome of the replication of the CPD lesion containing DNA section, allele specific qPCR primers were used on the PCR product. The primer pairs used to distinguish double stranded DNA templates after CPD lesion bypass were: GATTTAGAATTGTCCACCTCGCC and CCTTACTGCATGGATCGAGCT for GC at the site of both lesions, GATTTAGAATTGTCCACCTCGCC and TCCCTTACTGCATGGATCGATTT for AA insertion by TLS at the downstream lesion or TTTTGATTTAGAATTGTCCACCTCTT and CCTTACTGCATGGATCGAGC for AA insertion by TLS at the upstream lesion (the allele specific bases are underlined). These primers provide 1.000-100.000 fold enrichment over templates with the alternative sequence variants.

\subsection{Flow cytometry, cell cycle analysis and western blotting}

DT40 cells were UV-irradiated, labelled with bromodeoxyuridine, fixed and prepared for cell cycle analysis exactly as described [36], and analysed using a FACSCanto II instrument and FACSDiva 6.1 software (BD Biosciences). EGFP expression was measured in live cells using the same instrument.

For Western blot analysis, whole cell extracts of DT40 cells were fractionated by SDS-PAGE and transferred to a PVDF membrane using wet-transfer. After blocking in Tris-buffered saline (TBS) containing 5\% dry nonfat milk for $1 \mathrm{~h}$, the membrane was incubated with anti-PCNA (PC10, Abcam ab29, dilution 1:20000) at $4{ }^{\circ} \mathrm{C}$ overnight. Blots were washed and incubated with anti-mouse (Sigma A9044, dilution 1:20000) secondary antibody for $1 \mathrm{~h}$, and developed with the ECL system (Clarity Western ECL Blotting Substrate, Bio-Rad Laboratories). Chemiluminescent imaging was performed with a ChemiDoc MP system and the band intensities were quantified using the ImageLab software (Bio-Rad Laboratories). For the analysis of in vitro ubiquitylation, blots were blocked in TBS complemented with $5 \%$ bovine serum albumin, then incubated with anti-ubiquitin primary antibody (Abcam ab7780, dilution 1:1000) in TBS with $3 \%$ bovine serum albumin for $3.5 \mathrm{~h}$ at room temperature.

\subsection{Whole genome sequencing and mutation calling}

For the mutagenesis experiment, a single cell starting clone was isolated from each cell line and expanded, then four rounds of MMS (Sigma) treatments were performed in weekly intervals. One million cells were treated each time for one hour. Following the last treatment, single cell clones were isolated and expanded to two million cells prior to genomic DNA preparation using the Gentra Puregene Cell Kit (Qiagen).

Library preparation used the TruSeq DNA Nano Library Preparation Kit (Illumina) or the NEBNext Ultra DNA Library Prep Kit for Illumina (New England Biolabs). Sequencing was done on Illumina HiSeq 2500 v4 instruments $(2 \times 125$ bp PE). Library preparation and DNA sequencing was done at the Research Technology Support Facility of Michigan State University, USA, and at Novogene, Beijing, China. The reads were aligned to the chicken (Gallus gallus) reference sequence Galgal4.73, duplicate reads were removed and the aligned reads were realigned near indels as described [37].

Independently arising SNVs and short indels were identified using the IsoMut method developed for multiple isogenic samples [38]. Data from 40 different sequenced DT40 clones was used at this step, which included the samples presented in this article. Briefly, after applying a base quality filter of 30, data from all samples were compared at each genomic position, and filtered using optimised parameters of minimum mutated allele frequency $(0.2)$, minimum coverage of the mutated sample (5), and minimum reference allele frequency of all the other samples (0.93), and also filtered using a probability-based quality score calculated from the mutated sample and one other sample with the lowest reference allele frequency. The quality score threshold was set such that no more than five false positive SNVs would be detected in pre-treatment starting clones. Raw sequence data has been deposited with the European Nucleotide Archive under study accession number ERP019364.

\section{Results}

\subsection{Model for mimicking the monoubiquitylated form of PCNA}

To analyse and separate the phenotype of mono- and K63-linked polyubiquitylated PCNA in vivo, we fused wild type or K63R mutant versions of ubiquitin to the carboxy terminus of the non-ubiquitylable K164R mutant form of chicken PCNA. The two C-terminal glycine residues were removed from ubiquitin in the fusion constructs to prevent further modifications [25]. Alongside these $\mathrm{PCNA}^{\mathrm{K} 164 \mathrm{R}}-\mathrm{Ub}$ and PCNA ${ }^{\mathrm{K} 164 \mathrm{R}}-\mathrm{Ub}^{\mathrm{K} 63 \mathrm{R}}$ fusion constructs two further expression constructs were made that contained the wild type or K164R mutant PCNA as positive and negative controls (Fig. 1A). These constructs also express EGFP as part of a bicistronic message with an internal ribosome entry site (IRES) to indicate the expression level.

The PCNA fusion constructs were stably transfected into the DT40 $P C N A^{K 164 R}$ cell line in which the endogenous ubiquitylation/sumoylation site of PCNA is eliminated [33], therefore the effect of ubiquitylation is only from the fusion. Unlike the wild type ubiquitin fusion, the fused $\mathrm{Ub}^{\mathrm{K} 63 \mathrm{R}}$ cannot be polyubiquitylated through lysine 63, allowing us to genetically test the requirement for K63-linked polyubiquitylation. The expression levels of the constructs were carefully matched via EGFP expression and anti-PCNA Western blots (Fig. 1B, C). The amounts of the fusion proteins in these cell lines were approximately similar to the endogenous expression level of PCNA, indicating a 2-fold overexpression of PCNA in the transfected lines. We were unable to detect polyubiquitylated forms of either the endogenous PCNA or the fusion constructs in cell extracts by Western blot, therefore our studies must be considered primarily genetic, correlating phenotypic changes with the losses of potential PCNA post-translational modifications. To explore this issue further, we set up an in vitro ubiquitylation assay with human E1 (UBA1), E2 (UBC13-MMS2) and E3 (HLTF) enzymes, which have been shown to ubiquitylate PCNA that is monoubiquitylated by RAD6 and RAD18 [10]. We detected a band consistent with the ubiquitylation of PCNA ${ }^{\mathrm{K} 164 \mathrm{R}}$-Ub, which was absent when we used the $\mathrm{PCNA}^{\mathrm{K} 164 \mathrm{R}}-\mathrm{Ub}^{\mathrm{K} 63 \mathrm{R}}$ substrate (Fig. 1D). The formation of this modification was dependent on the E1 and E2 enzymes, and slightly enhanced by the presence of HLTF. Interestingly, we did not detect longer ubiquitin chains, possibly suggesting that the HLTF preparation had low activity. Although it has been suggested that PCNA polyubiquitylation takes place through the addition of pre-assembled polyubiquitin chains [39], we conclude from these results that the $\mathrm{PCNA}^{\mathrm{K} 164 \mathrm{R}_{-} \mathrm{Ub}}$ fusion protein can be further ubiquitylated by UBC13-MMS2 in the absence of RAD18 in a manner dependent on lysine 63 of ubiquitin. This suggests that the PCNA-Ub fusion protein is a suitable substrate for K63-linked polyubiquitylation, and provides support for the argument that polyubiquitin chains cannot form on the $\mathrm{PCNA}^{\mathrm{K} 164 \mathrm{R}}-\mathrm{Ub}^{\mathrm{K} 63 \mathrm{R}}$ protein.

\subsection{The role of PCNA modifications in UV damage tolerance}

PCNA modifications on K164 are required for the unhindered bypass of the two most common UV photoproducts [20,36]. To test whether the forced ubiquitylation of PCNA in the cell lines that express PCNA-Ub fusions can substitute for these modifications, we first measured cell cycle progression without or with UV irradiation (Fig. 2). Untreated populations had similar cell cycle distribution, so without DNA damaging treatment, there appear to be no differences between the newly generated cell lines (Fig. 2B). After low-dose UV irradiation and $6 \mathrm{~h}$ recovery there was a marked increase in cells arrested in S phase in all cell lines (Fig. 2C), but after $24 \mathrm{~h}$ recovery the cell cycle profile of the wild type cells was very similar to that of the 
A

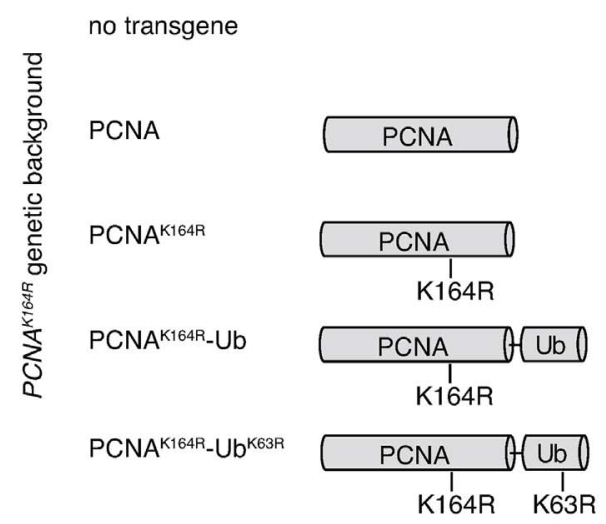

B

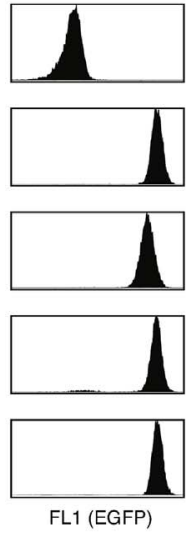

C

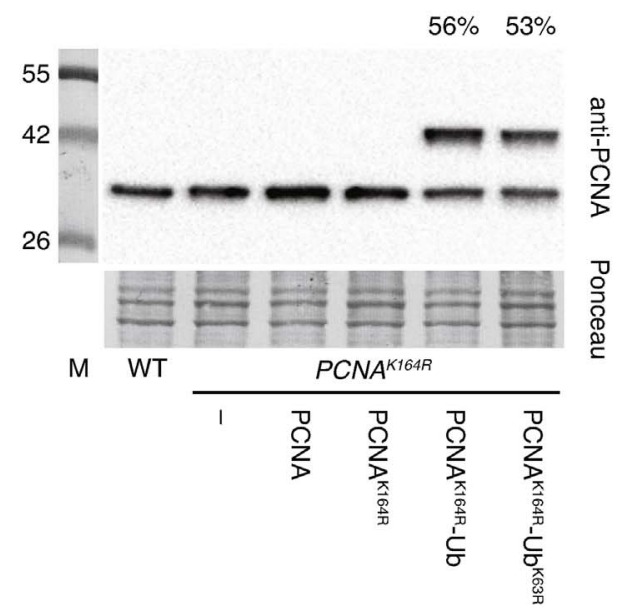

D
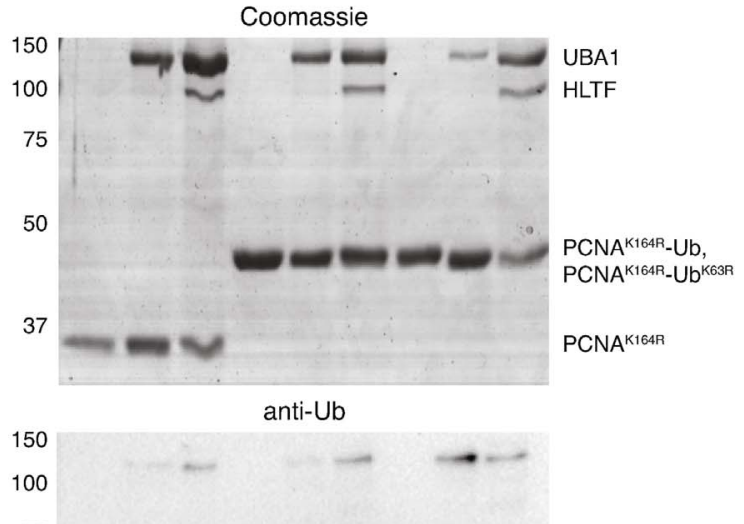

75

50

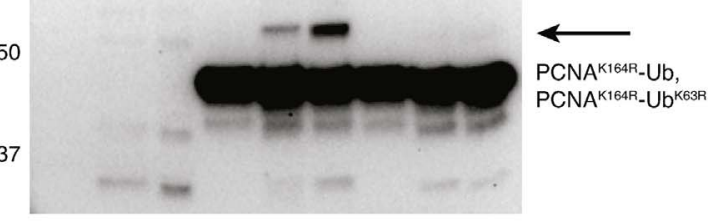

-++-++-++ UBA 1

-++-++-++ UBC13-MMS2

$--+--+-\quad+$ HLTF

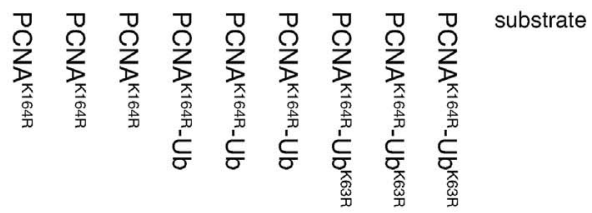

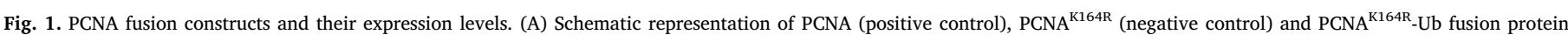

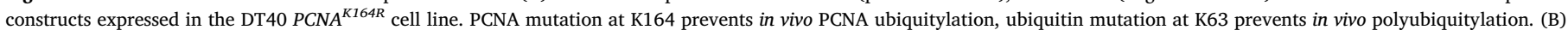

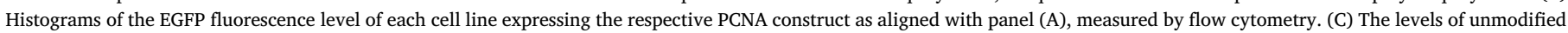

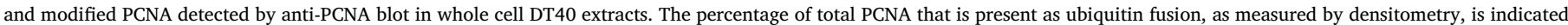

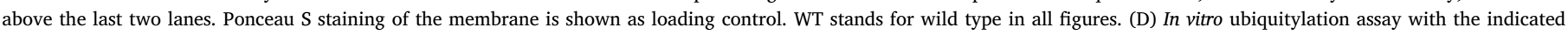

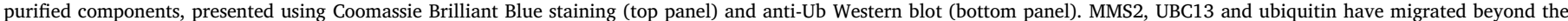
bottom of the gel. The arrow indicates a ubiquitylated form of the PCNA ${ }^{\mathrm{K} 164 \mathrm{R}}-\mathrm{Ub}$ fusion protein. Size markers are in kDa.

untreated cells, with a slight increase in the proportion of cells arrested in $S$ phase (Fig. 2D). In contrast, there was a marked increase in G2/M phase cells and a reduction of G1 and $\mathrm{S}$ phase cells in the $P C N A^{K 164 R}$ cell line. The expression of wild type PCNA rescued this defect, while the expression of the mutated $\mathrm{PCNA}^{\mathrm{K} 164 \mathrm{R}}$ protein did not. There was a clear, though partial rescue of the UV-induced cell cycle delay in the cell lines expressing the $\mathrm{PCNA}^{\mathrm{K} 164 \mathrm{R}}-\mathrm{Ub}$ or $\mathrm{PCNA}^{\mathrm{K} 164 \mathrm{R}}-\mathrm{Ub}^{\mathrm{K} 63 \mathrm{R}}$ fusion proteins, in which $38-39 \%$ of the cells were in S phase $24 \mathrm{~h}$ after UV exposure, with no significant difference between them (Fig. 2D). The proportion of $S$ phase cells in the $\mathrm{PCNA}^{\mathrm{K} 164 \mathrm{R}}-\mathrm{Ub}^{\mathrm{K} 63 \mathrm{R}}$ expressing line was not significantly different from the wild type cell line ( $\mathrm{p}=0.09, t$-test), but significantly lower than the rescue line that expresses wild type PCNA $(\mathrm{p}=0.0002)$ and significantly higher than the $P C N A^{K 164 R}$ and $P C N A^{K 164 R}: \mathrm{PCNA}^{\mathrm{K} 164 \mathrm{R}}$ negative controls $(\mathrm{p}<0.0001$ and $\mathrm{p}=0.0010$, respectively; due to multiple comparisons, the significance limit should be lowered to $p=0.01$ in this set of tests). This indicates that the single C-terminally fused ubiquitin with or without further potential K63linked ubiquitin modifications can equally substitute for PCNA modifications on $\mathrm{K} 164$.

To complement the cell cycle analysis, we measured the UV sensitivity of the cell lines using colony formation assays. Compared to the wild type, the $P C N A^{K 164 R}$ and $P C N A^{K 164 R}: \mathrm{PCNA}^{\mathrm{K} 164 \mathrm{R}}$ negative control cells were hypersensitive to UV, while the cell lines that express the $\mathrm{PCNA}^{\mathrm{K} 164 \mathrm{R}}-\mathrm{Ub}$ and $\mathrm{PCNA}^{\mathrm{K} 164 \mathrm{R}}-\mathrm{Ub}^{\mathrm{K} 63 \mathrm{R}}$ fusion proteins showed similar UV sensitivity to the wild type and the $P C N A^{K 164 R}$ :PCNA rescue cell line (Fig. 3A). This suggests that the potential polyubiquitylation in the PCNA ${ }^{\mathrm{K} 164 \mathrm{R}}$-Ub expressing cell line is not required for cell survival following UV irradiation.

\subsection{PCNA monoubiquitylation is required for efficient translesion synthesis over a UV lesion}

We next investigated the influence of PCNA modifications on the choice of damage bypass mechanism using a shuttle plasmid assay that can measure the ratio of TLS and error-free bypass that uses the complementary strand as template $[20,36]$. In order to investigate DNA damage bypass only, we eliminated the primary repair system of UV lesions by creating a nucleotide excision repair deficient, $X P A^{-}$knockout genetic background in each cell line. We investigated the replication products of $\mathrm{PQ1}-\mathrm{CPDs}$ plasmids that contained one T-T CPD lesion on each strand in a staggered arrangement, separated by $28 \mathrm{bp}$, and opposed by a mismatched GC dinucleotide [36]. The overall proportion of replicated plasmids, calculated from the DpnI resistance of short plasmid fragments, was in the $2.2-4.9 \%$ range across all the samples, with no significant differences found by ANOVA (Fig. 2B). Allelespecific primers were used in quantitative PCR reactions to measure the relative quantities of the main sequence variants at the lesions among replication products. The insertion of AA opposite either lesion 
A

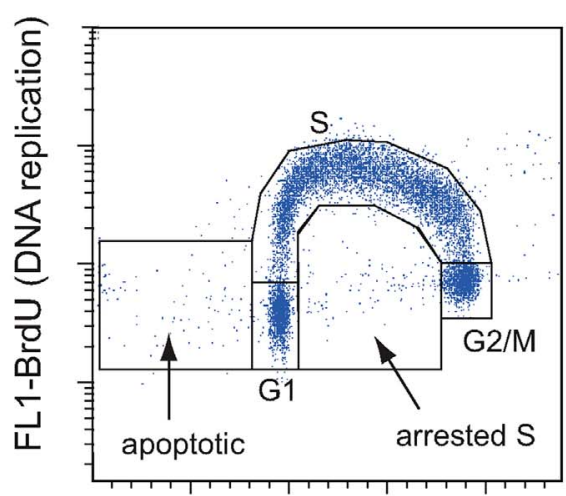

FL3-PI (DNA content)

B

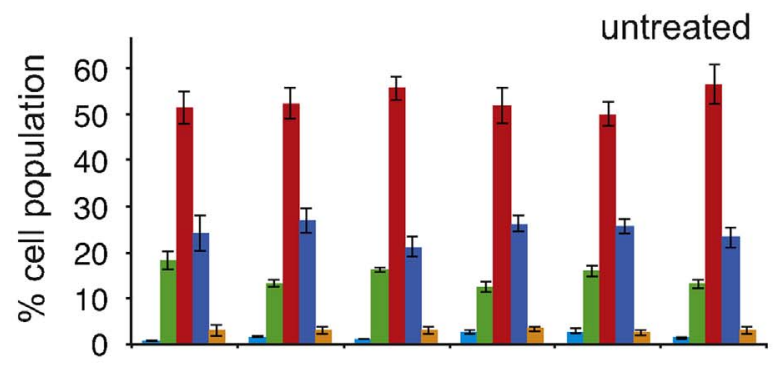

C

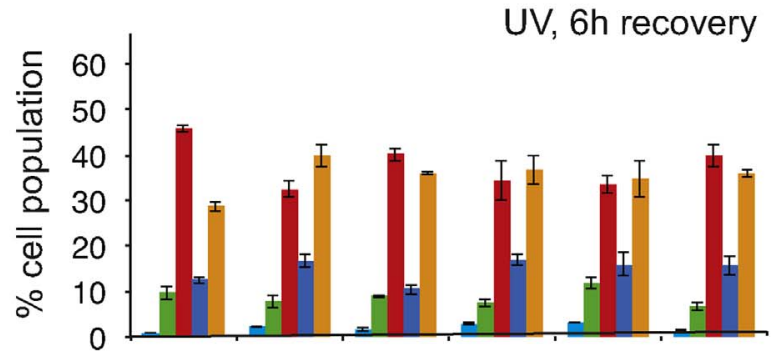

D

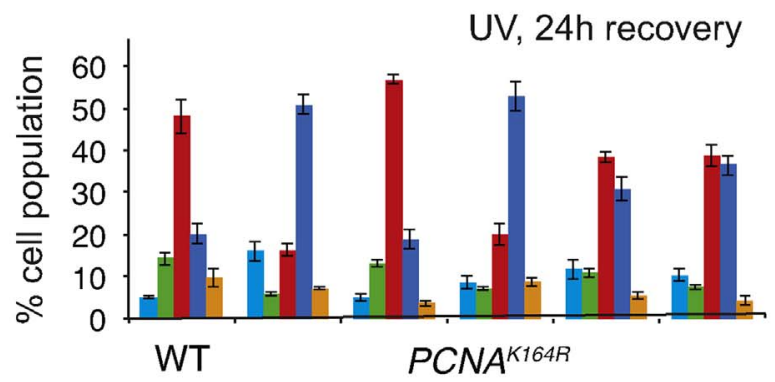

Fig. 2. Cell cycle analysis. The DNA content of DT40 cells of the indicated genotypes were analysed using propidium iodide staining (horizontal axis), and the rate of DNA replication using anti-BrdU-FITC antibody staining (vertical axis). (A) The rationale for assigning cell populations to different cell cycle phases, an apoptotic category with sub-G1 DNA content, and an arrested $S$ phase category for non-replicating cells with an S phase DNA content. (B-D) The percentage of cells in each category. Cell cycle phases were measured in untreated cells (B) or following $1 \mathrm{~J} / \mathrm{m}^{2} \mathrm{UV}$ irradiation and $6 \mathrm{~h}$ (C) or $24 \mathrm{~h}$ recovery (D). The mean and S.E.M of five (three for the $6 \mathrm{~h}$ time point) independent experiments is shown.

indicates TLS, while the presence of GC at the site of both lesions in the replication product was taken as evidence of error-free bypass by template switching (Fig. 4A). The rare mutagenic TLS outcomes such as TA were not measured, which is expected to exclude $11-17 \%$ of all TLS events from our analysis [36]. In $X P A^{-}$cells $61 \%$ of successful bypass products bore evidence of TLS, whereas in $X P A^{-} P C N A^{K 164 R}$ we observed a modest but significant reduction $(\mathrm{p}=0.0284, t$-test $)$ in the proportion of TLS to a mean of $52 \%$ (Fig. 4C). These data, obtained with allele-specific qPCR, are in very good agreement with earlier data derived from the sequencing of individual replication products of the same experiment [36]. The reduced proportion of TLS confirms a role for PCNA ubiquitylation in the process, and the remaining PCNA ubiquitylation independent TLS is likely due to TLS polymerase recruitment through REV1 [40,20].

The expression of the non-ubiquitylable mutant PCNA ${ }^{\mathrm{K} 164 \mathrm{R}}$ did not rescue the TLS defect $(p=0.0166)$, whereas the transgenic expression of wild type PCNA fully restored the use of TLS for CPD bypass. Importantly, the expression of either PCNA-Ub fusion construct $\left(\mathrm{PCNA}^{\mathrm{K} 164 \mathrm{R}}-\mathrm{Ub}\right.$ and $\mathrm{PCNA}^{\mathrm{K} 164 \mathrm{R}}-\mathrm{Ub}^{\mathrm{K} 63 \mathrm{R}}$ ) also restored the proportion of TLS to near the $60 \%$ level (Fig. 4C). Thus the C-terminally fused ubiquitin can substitute for the requirement for PCNA ubiquitylation in the regulation of TLS. Moreover, the fusion of K63R-mutant ubiquitin provides the same rescue effect, even though it does not allow the formation of polyubiquitin chains. We can conclude that PCNA monoubiquitylation is the only PCNA modification that is required for the bypass of CPD lesions, and potential polyubiquitylation of PCNA does not influence the outcome through either TLS or the use of the sister chromatid as template.

3.4. The role of PCNA modifications in the bypass of cisplatin- and MMSinduced DNA damage

UV photoproducts comprise a specific set of well-studied DNA lesions. To assay the role of PCNA polyubiquitylation in the bypass of a wider variety of DNA lesions, we treated cells with MMS, an alkylating agent [41], or cisplatin, which generates intrastrand and interstrand crosslinks [42]. The PCNA ${ }^{K 164 R}$ cell line and the PCNA ${ }^{\mathrm{K} 164 \mathrm{R}_{-}}$ expressing negative control were hypersensitive to both MMS and cisplatin, and the expression of wild type PCNA in the PCNA ${ }^{K 164 R}$ background fully restored sensitivity to the wild type level (Fig. 3B, C). In case of MMS, the expression of either the PCNA ${ }^{\mathrm{K} 164 \mathrm{R}}-\mathrm{Ub}$ or the $\mathrm{PCNA}^{\mathrm{K} 164 \mathrm{R}}-\mathrm{Ub}^{\mathrm{K} 63 \mathrm{R}}$ fusion proteins also fully rescued the hypersensitivity, thus the monoubiquitin modification was sufficient to support a wild type MMS response. The expression of $\mathrm{PCNA}^{\mathrm{K} 164 \mathrm{R}}-\mathrm{Ub}$ or $\mathrm{PCNA}^{\mathrm{K} 164 \mathrm{R}}-\mathrm{Ub}^{\mathrm{K} 63 \mathrm{R}}$ resulted in similar partial rescue of the cisplatin sensitivity of the $P C N A^{K 164 R}$ cell line (see discussion).

To further test whether the reduced sensitivity upon the expression of PCNA-ubiquitin fusions is not due to the increase in the overall amounts of PCNA in the cell, we created cell lines expressing matched

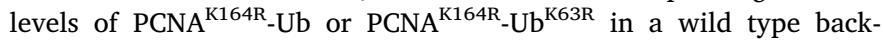
ground. We found that the presence of endogenous and transgenic PCNA together (doubling the amount of endogenous PCNA) did not change MMS sensitivity (Fig. 3D), demonstrating the specificity of rescuing effect of the fusion proteins in the $P C N A^{K 164 R}$ background.

\subsection{Monoubiquitylated PCNA is sufficient to restore the mutation spectrum}

An important consequence of TLS is the potential generation of somatic mutations, in contrast with error-free lesion bypass using an alternative template. Interference with PCNA ubiquitylation-dependent template switching is therefore expected to increase the rate of mutagenesis. Whole genome sequencing allows the detection of all genomic mutations that arise during the course of an experiment, and we recently employed this strategy to demonstrate a high rate of mutagenesis in response to MMS treatment, with the predominance of $\mathrm{T}>\mathrm{A}$ and $\mathrm{C}>$ A changes amongst base substitution mutations [37]. 


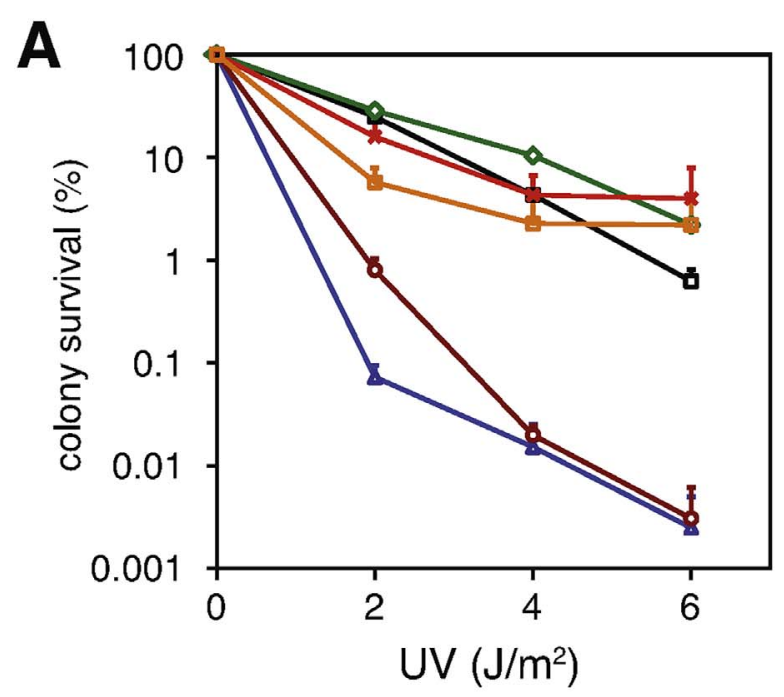

$$
\begin{aligned}
& \rightarrow \text { WT } \\
& \triangle P C N A^{K 164 R} \\
& \text {-o PCNA }{ }^{K 164 R: P C N A} \\
& \text {-o- PCNA }{ }^{K 164 R: P C N A}{ }^{K 164 R} \\
& \text { * PCNA }{ }^{K 164 R: P C N A} A^{K 164 R-U b} \\
& \because P C N A^{K 164 R: P C N A}{ }^{\mathrm{K} 164 \mathrm{R}}-\mathrm{Ub}^{\mathrm{K} 63 \mathrm{R}}
\end{aligned}
$$

B

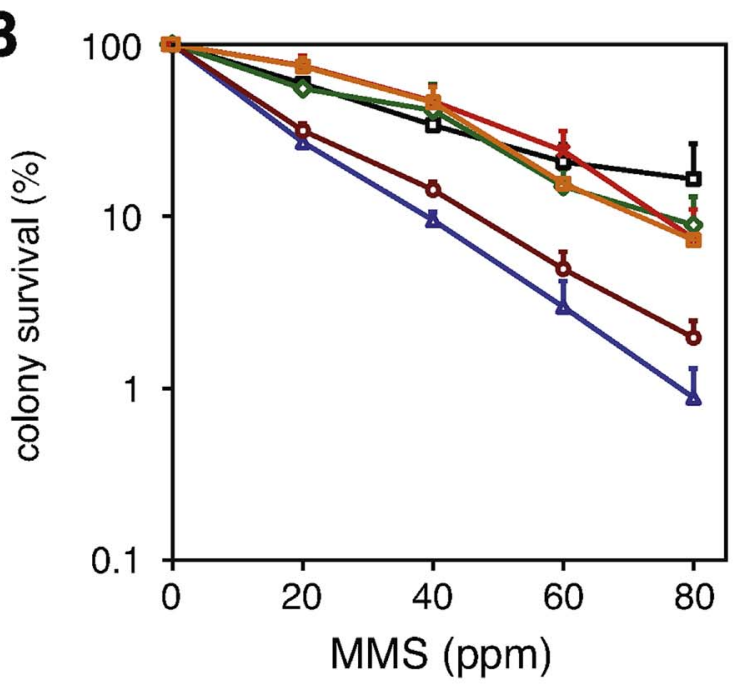

D

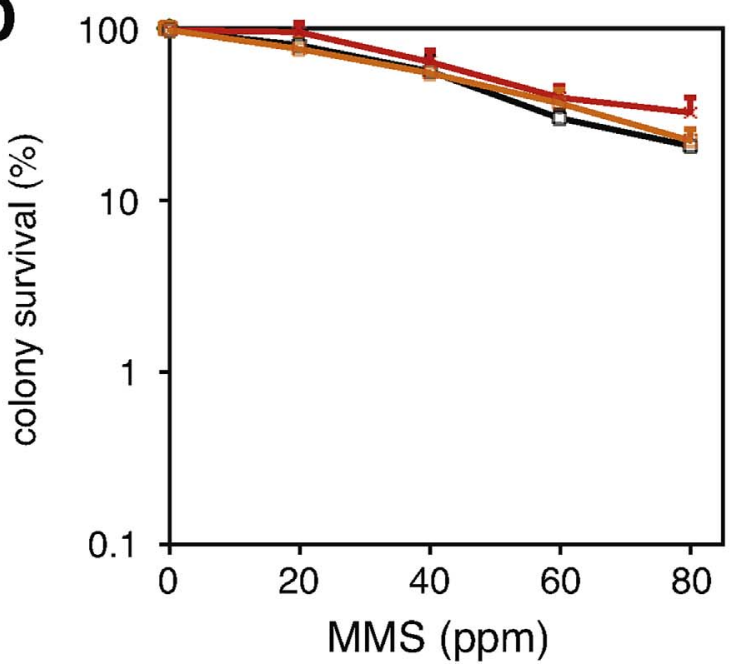

C

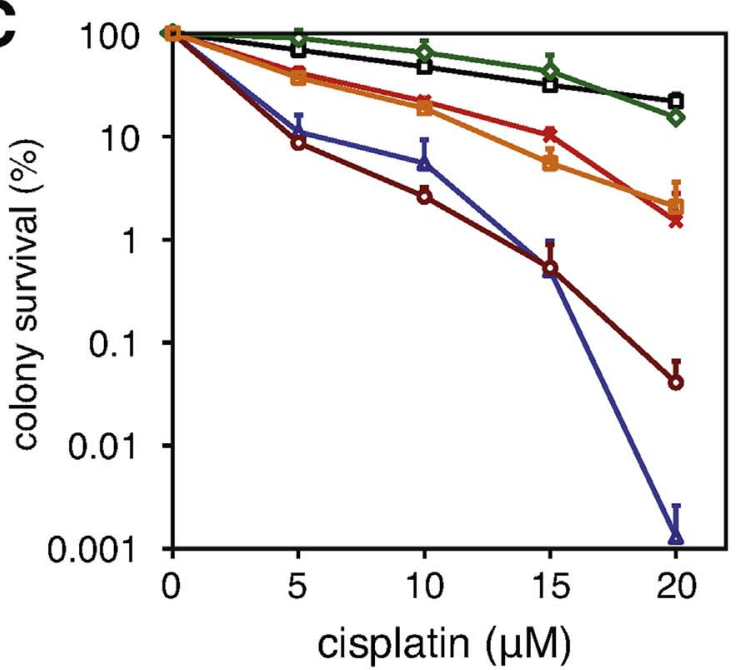

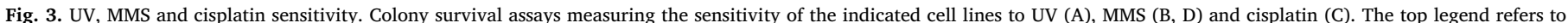
panels (A-C). The mean of three independent experiments is shown, error bars indicate S.E.M. 
A

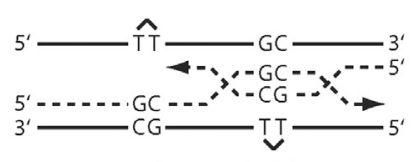

template switching

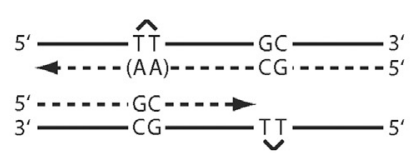

TLS on top strand

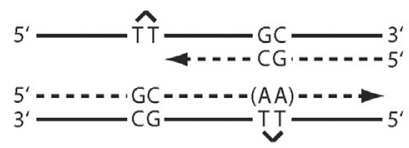

TLS on bottom strand
B

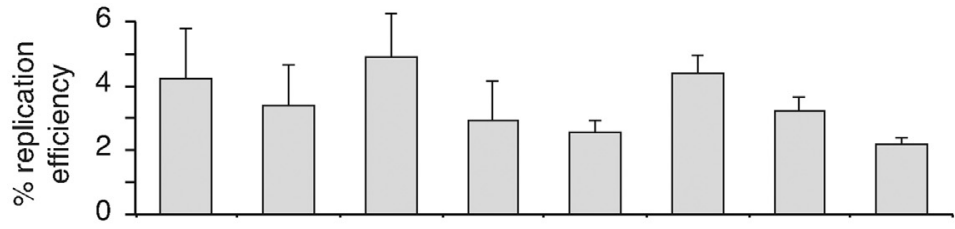

C

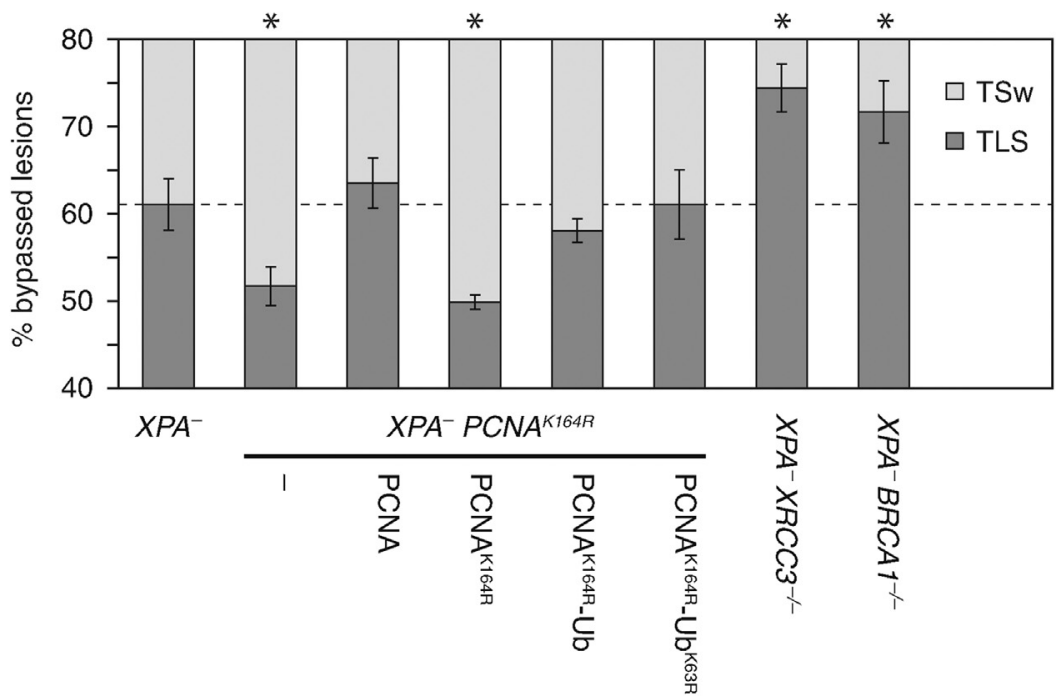

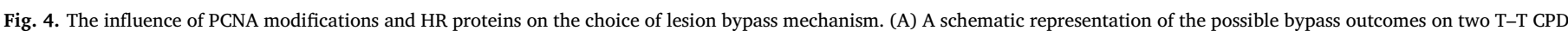

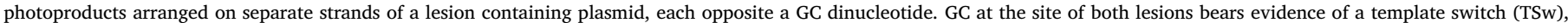

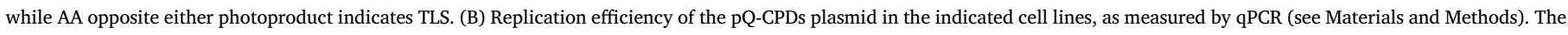

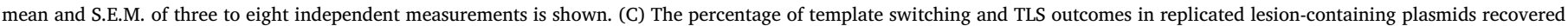
from the indicated cell lines, measured using allele-specific qPCR. The mean and S.E.M. of four to six independent measurements is shown.
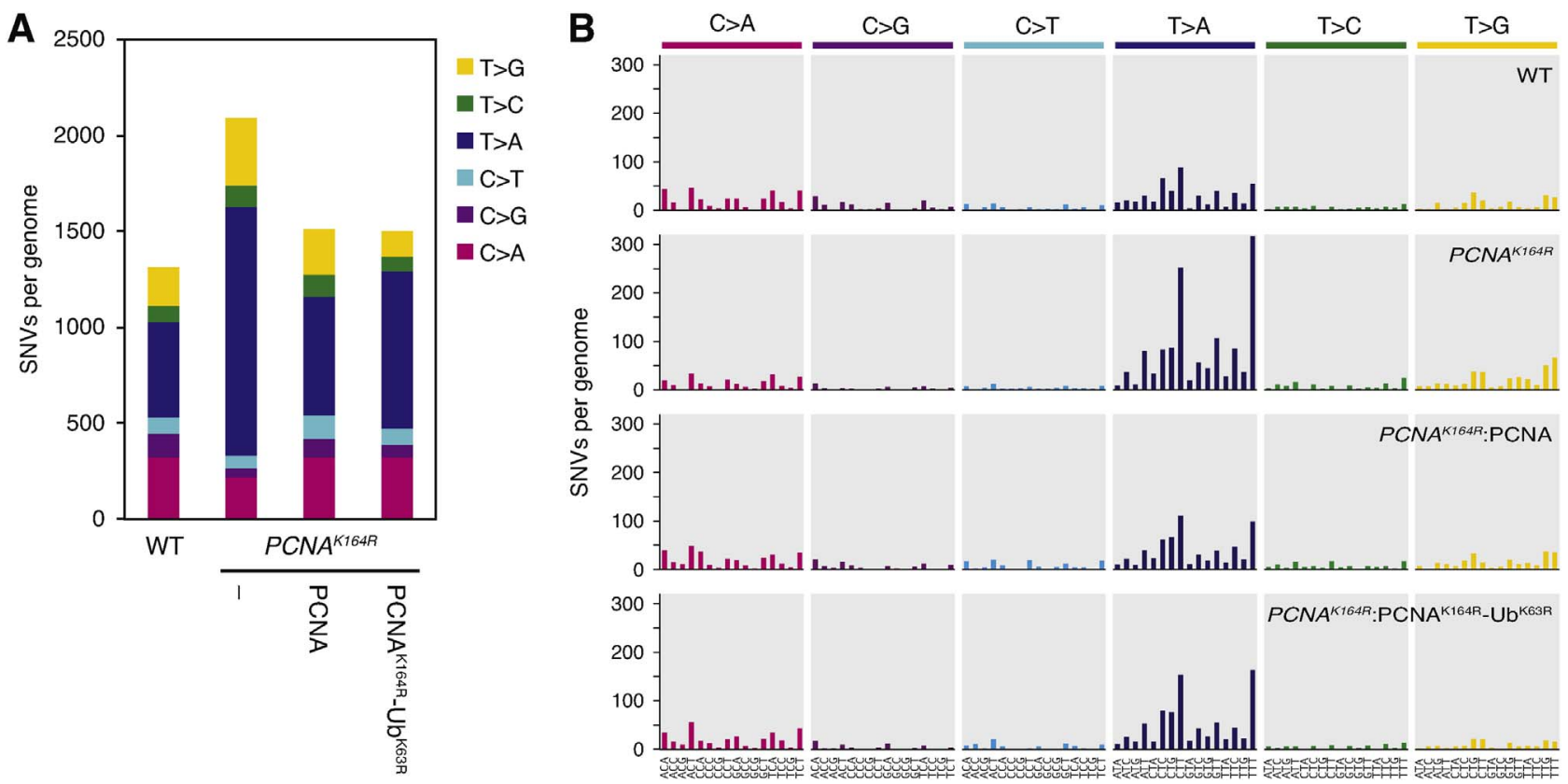

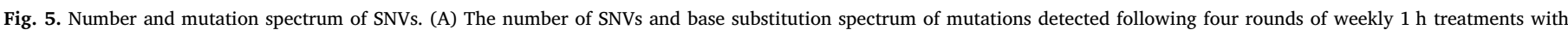

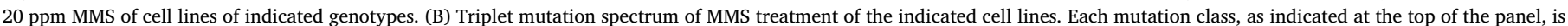
separated into 16 categories based on the identity of the preceding and following nucleotide as shown at the bottom.

To use MMS-induced mutations as a background for measuring the mutagenic impact of PCNA modifications, we repeatedly subjected single cell clones to one-hour treatments with $20 \mathrm{ppm}(236 \mu \mathrm{M}) \mathrm{MMS}$, followed by the isolation of post-treatment single cell clones. The identification of unique mutations in cell clones isolated from the treated populations indicated increased base substitution mutagenesis in the $P C N A^{K 164 R}$ cell line compared to the wild type (Fig. 5A), with an especially noticeable 2.6-fold increase in the number of $\mathrm{T}>\mathrm{A}$ mutations, primarily in the context of further pyrimidine bases (Fig. 5A, B). The expression of wild type PCNA nearly rescued this phenotype, reducing both the overall number of SNVs and specifically the $\mathrm{T}>\mathrm{A}$ mutations. This reduction in mutagenesis in the presence of wild type PCNA cannot be the consequence of PCNA polyubiquitylation-dependent template switching, as the expression of the PCNA ${ }^{\mathrm{K} 164 \mathrm{R}_{-}}$ 
$\mathrm{Ub}^{\mathrm{K} 63 \mathrm{R}}$ fusion protein similarly reduced SNV numbers and nearly restored the wild type mutation spectrum (Fig. 5A, B). If, based on their identical MMS sensitivity we considered results from the wild type and the two rescued cell line samples as belonging to the same distribution, the $P C N A^{K 164 R}$ sample would fall outside the $95 \%$ confidence interval for both the overall number of SNVs and the $\mathrm{T}>$ A mutations. The increased mutagenesis in the $P C N A^{K 164 R}$ cell line may be due to mutagenic REV1-dependent TLS, as shown in the case of UV CPD lesions [36].

\subsection{Template switching bypass uses a recombinational mechanism}

The proportion of bypass events with a template switch outcome was unaffected in the $P C N A^{K 164 R}: \mathrm{PCNA}^{\mathrm{K} 164 \mathrm{R}}-\mathrm{Ub}^{\mathrm{K} 63 \mathrm{R}}$ mutant (Fig. 4C), arguing against an involvement of PCNA polyubiquitylation in template switching bypass. To test whether the observed use of the sister chromatid as template requires HR factors, we created $X P A^{-} B R C A^{-\prime}$ - and $X P A^{-} X R C C 3^{-/-}$cell lines. BRCA1 is a critical factor of HR, playing roles in controlling the resection of broken double stranded DNA ends [43] and in the recruitment of the essential HR protein RAD51 [44,45], while XRCC3 is a RAD51 paralogue that is also required for RAD51 recruitment [46]. When comparing to the $X P A^{-}$ cell line, allele-specific qPCR analysis of replicated pQ1-CPDs plasmids revealed a significant increase in the proportional use of TLS from $61 \%$ to $74 \%$ and $72 \%$ due to the disruption of XRCC3 and BRCA1 respectively, with a corresponding decrease of template switching outcomes (Fig. 4C, $\mathrm{p}=0.006$ and $\mathrm{p}=0.0428, t$-test). The disappearance of the apparent template switch events suggests that the use of the sister chromatid as a template during lesion bypass is in large part a BRCA1-dependent, HR-related mechanism.

\section{Discussion}

In this study we made use of genetic experiments in the chicken DT40 cell line to probe the function of PCNA modifications in DNA damage tolerance and lesion bypass. The fusion of a single ubiquitin to PCNA rescued the damage sensitivity of a non-ubiquitylable PCNA mutant, and also restored the rate of TLS over CPD UV photoproducts. The expression of a PCNA-ubiquitin fusion variant that prevented the addition of further ubiquitin units showed identical rescuing phenotypes, demonstrating a lack of requirement for PCNA polyubiquitylation in UV and MMS-induced DNA damage tolerance.

The conserved K164 residue of PCNA is a platform for different modifications: mono- and polyubiquitylation, sumoylation. Researchers have been able to specifically test the function of PCNA ubiquitylation using PCNA-ubiquitin fusion proteins, which partially rescued damage sensitivity in $S$. cerevisiae Rad18 mutants $[25,26]$, or TLS in $S$. pombe pcn1-K164R mutants [27]. In contrast, we observed a full rescue of UV and MMS damage sensitivity and TLS in $P C N A^{K 164 R}$ mutant vertebrate cells which offered the opportunity to specifically test the requirement for PCNA mono- or polyubiquitylation. The full rescue suggests that TLS polymerases are able to bind C-terminal PCNA-Ub fusion proteins. Though siRNA silencing based experiments in human cells suggested that Pol $\eta$ was not required for DNA damage tolerance conferred by PCNA-Ub fusions [28], we previously observed an identical requirement for PCNA ubiquitylation and Pol $\eta$ in the bypass of CPD UV photoproducts [36], and in this study the same wild type level of TLS was achieved by the monoubiquitylation-equivalent $\mathrm{Ub}^{\mathrm{K} 63 \mathrm{R}}$ fusion, suggesting that at CPD lesions the main function of PCNA ubiquitylation is the recruitment of $\mathrm{Pol} \eta$.

TLS is not fully dependent on PCNA ubiquitylation [40,20], which is concordant with the increased rate of mutagenesis we observed in the genome of MMS-treated $P C N A^{K 164 R}$ cells, which is likely due to mutagenic TLS in the absence of PCNA ubiquitylation. The reduction of the rate of mutagenesis and the restoration of the wild type mutagenic spectrum by the expression of the PCNA ${ }^{\mathrm{K} 164 \mathrm{R}}-\mathrm{Ub}^{\mathrm{K} 63 \mathrm{R}}$ fusion may then be explained by the recruitment of alternative, less mutagenic TLS polymerases. The only contexts in which we did not observe full rescue by 'monoubiquitylated' PCNA were sensitivity to cisplatin, and recovery from UV-induced cell cycle arrest. Here, the similar partial rescue by wild type or K63R mutant ubiquitin suggests that some further PCNA modification may be needed for damage tolerance. A candidate for such modification is PCNA SUMOylation, which is not possible at residue K164 in the cell lines that express the PCNA-Ub fusion proteins, though it may be necessary to regulate HR during the repair or bypass of cisplatin lesions $[47,48]$. An alternative explanation one must consider for the observed imperfect rescue is that the $\mathrm{PCNA}^{\mathrm{K} 164 \mathrm{R}}$-Ub fusion may not be a perfect mimic for PCNA monoubiquitylated on K164 with regards to cisplatin survival, or, if the $\mathrm{PCNA}^{\mathrm{K} 164 \mathrm{R}}$-Ub fusion cannot be properly polyubiquitylated, this might demonstrate a requirement for polyubiquitylation under these circumstances.

The main conclusion from our results is the lack of a detectable requirement for PCNA polyubiquitylation. We did not physically detect polyubiquitylation of the expressed $\mathrm{PCNA}^{\mathrm{K} 164 \mathrm{R}}$-Ub fusion protein in vivo. However, regardless of whether this can take place, the full rescue of several PCNA ${ }^{\mathrm{K} 164 \mathrm{R}}$ phenotypes by both PCNA-Ub fusions provides a genetic demonstration that monoubiquitylation was sufficient for tolerance to UV and MMS, bypass of CPD UV photoproducts, and the avoidance of MMS-induced genomic mutations.

Post-translational modification of PCNA with two or more ubiquitin units has been observed in multiple experimental systems, including the DT40 cells used in this study [49], even though HLTF is absent from the chicken genome. What may then be the function of PCNA polyubiquitylation? The structure specific helicase activity of Rad5 and its orthologues HLTF and SHPRH provided the best lead for a function in replication fork reversal, which is a potential mechanism for nonmutagenic template switching bypass. However, HLTF and SHPRH have damage-specific and rather complex effects on point mutations, mostly due to their influence on TLS [22], while a template switching role would predict a clear anti-mutagenic function. The ZRANB3 protein can bind polyubiquitylated PCNA and promote replication fork remodelling and restart [50], and emerging evidence suggests a distinct role for fork reversal in the protection of stalled replication forks [51,52]. A role for PCNA polyubiquitylation in fork reversal and stabilisation would explain the increased rate of chromosome breakage due to the loss of SHPRH or HLTF $[24,53]$, and could also explain the incomplete rescue of late $\mathrm{S} / \mathrm{G} 2$ phase arrest we observed with the $\mathrm{PCNA}^{\mathrm{K} 164 \mathrm{R}}-\mathrm{Ub}^{\mathrm{K} 63 \mathrm{R}}$ fusion.

In conclusion, our dissection of PCNA post-translational modifications in the DT40 genetic model suggests there is no requirement for PCNA polyubiquitylation in DNA damage tolerance and error-free damage bypass.

\section{Conflict of interest}

The authors declare that there are no conflicts of interest.

\section{Acknowledgments}

We thank Ádám Póti for his assistance in data processing, György Várady for help with flow cytometry, and Lajos Haracska for the HLTF cDNA. This study was supported by Momentum Grant LP2011-015 of the Hungarian Academy of Sciences.

\section{References}

[1] G. Maga, U. Hubscher, Proliferating cell nuclear antigen (PCNA): a dancer with many partners, J. Cell Sci. 116 (2003) 3051-3060.

[2] G.L. Moldovan, B. Pfander, S. Jentsch, PCNA, the maestro of the replication fork, Cell 129 (2007) 665-679.

[3] J.T. Fox, K.Y. Lee, K. Myung, Dynamic regulation of PCNA ubiquitylation/ deubiquitylation, FEBS Lett. 585 (2011) 2780-2785. 
[4] C. Hoege, B. Pfander, G.L. Moldovan, G. Pyrowolakis, S. Jentsch, RAD6-dependent DNA repair is linked to modification of PCNA by ubiquitin and SUMO, Nature 419 (2002) 135-141.

[5] L.J. Simpson, A.L. Ross, D. Szuts, C.A. Alviani, V.H. Oestergaard, K.J. Patel, J.E. Sale, RAD18-independent ubiquitination of proliferating-cell nuclear antigen in the avian cell line DT40, EMBO Rep. 7 (2006) 927-932.

[6] M. Bienko, C.M. Green, N. Crosetto, F. Rudolf, G. Zapart, B. Coull, P. Kannouche G. Wider, M. Peter, A.R. Lehmann, et al., Ubiquitin-binding domains in Y-family polymerases regulate translesion synthesis, Science 310 (2005) 1821-1824.

[7] P.L. Kannouche, J. Wing, A.R. Lehmann, Interaction of human DNA polymerase eta with monoubiquitinated PCNA; a possible mechanism for the polymerase switch in response to DNA damage, Mol. Cell 14 (2004) 491-500.

[8] B.S. Plosky, A.E. Vidal, A.R. Fernandez de Henestrosa, M.P. McLenigan, J.P. McDonald, S. Mead, R. Woodgate, Controlling the subcellular localization of DNA polymerases iota and eta via interactions with ubiquitin, EMBO J. 25 (2006) $2847-2855$.

[9] D. Branzei, M. Seki, T. Enomoto, Rad18/Rad5/Mms2-mediated polyubiquitination of PCNA is implicated in replication completion during replication stress, Genes Cells 9 (2004) 1031-1042.

[10] I. Unk, I. Hajdu, K. Fatyol, J. Hurwitz, J.H. Yoon, L. Prakash, S. Prakash, L. Haracska, Human HLTF functions as a ubiquitin ligase for proliferating cell nuclear antigen polyubiquitination, Proc. Natl. Acad. Sci. U. S. A. 105 (2008) 3768-3773.

[11] I. Unk, I. Hajdu, K. Fatyol, B. Szakal, A. Blastyak, V. Bermudez, J. Hurwitz, L. Prakash, S. Prakash, L. Haracska, Human SHPRH is a ubiquitin ligase for Mms2Ubc13-dependent polyubiquitylation of proliferating cell nuclear antigen, Proc. Natl. Acad. Sci. U. S. A. 103 (2006) 18107-18112.

[12] H. Zhang, C.W. Lawrence, The error-free component of the RAD6/RAD18 DNA damage tolerance pathway of budding yeast employs sister-strand recombination, Proc. Natl. Acad. Sci. U. S. A. 102 (2005) 15954-15959.

[13] D. Branzei, F. Vanoli, M. Foiani, SUMOylation regulates Rad18-mediated template switch, Nature 456 (2008) 915-920.

[14] E.C. Minca, D. Kowalski, Multiple Rad5 activities mediate sister chromatid recombination to bypass DNA damage at stalled replication forks, Mol. Cell 38 (2010) 649-661.

[15] J. Atkinson, P. McGlynn, Replication fork reversal and the maintenance of genome stability, Nucleic Acids Res. 37 (2009) 3475-3492.

[16] N.P. Higgins, K. Kato, B. Strauss, A model for replication repair in mammalian cells, J. Mol. Biol. 101 (1976) 417-425.

[17] A. Blastyak, L. Pinter, I. Unk, L. Prakash, S. Prakash, L. Haracska, Yeast Rad5 protein required for postreplication repair has a DNA helicase activity specific for replication fork regression, Mol. Cell 28 (2007) 167-175.

[18] L.G. Ball, X. Xu, S. Blackwell, M.D. Hanna, A.D. Lambrecht, W. Xiao, The Rad5 helicase activity is dispensable for error-free DNA post-replication repair, DNA Repair (Amst.) 16 (2014) 74-83.

[19] L. Izhar, O. Ziv, I.S. Cohen, N.E. Geacintov, Z. Livneh, Genomic assay reveals tolerance of DNA damage by both translesion DNA synthesis and homologydependent repair in mammalian cells, Proc. Natl. Acad. Sci. U. S. A. 110 (2013) E1462-1469.

[20] D. Szuts, A.P. Marcus, M. Himoto, S. Iwai, J.E. Sale, REV1 restrains DNA polymerase zeta to ensure frame fidelity during translesion synthesis of UV photoproducts in vivo, Nucleic Acids Res. 36 (2008) 6767-6780.

[21] R.K. Chiu, J. Brun, C. Ramaekers, J. Theys, L. Weng, P. Lambin, D.A. Gray, B.G. Wouters, Lysine 63-polyubiquitination guards against translesion synthesisinduced mutations, PLoS Genet. 2 (2006) e116.

[22] J.R. Lin, M.K. Zeman, J.Y. Chen, M.C. Yee, K.A. Cimprich, SHPRH and HLTF act in a damage-specific manner to coordinate different forms of postreplication repair and prevent mutagenesis, Mol. Cell 42 (2011) 237-249.

[23] P.H. Krijger, K.Y. Lee, N. Wit, P.C. van den Berk, X. Wu, H.P. Roest, A. Maas, H. Ding, J.H. Hoeijmakers, K. Myung, et al., HLTF and SHPRH are not essential for PCNA polyubiquitination: survival and somatic hypermutation: existence of an alternative E3 ligase, DNA Repair (Amst.) 10 (2011) 438-444.

[24] A. Motegi, H.J. Liaw, K.Y. Lee, H.P. Roest, A. Maas, X. Wu, H. Moinova, S.D. Markowitz, H. Ding, J.H. Hoeijmakers, et al., Polyubiquitination of proliferating cell nuclear antigen by HLTF and SHPRH prevents genomic instability from stalled replication forks, Proc. Natl. Acad. Sci. U. S. A. 105 (2008) 12411-12416.

[25] J.L. Parker, A.B. Bielen, I. Dikic, H.D. Ulrich, Contributions of ubiquitin- and PCNAbinding domains to the activity of Polymerase eta in Saccharomyces cerevisiae, Nucleic Acids Res. 35 (2007) 881-889.

[26] L. Pastushok, M. Hanna, W. Xiao, Constitutive fusion of ubiquitin to PCNA provides DNA damage tolerance independent of translesion polymerase activities, Nucleic Acids Res. 38 (2010) 5047-5058.

[27] S. Ramasubramanyan, S. Coulon, R.P. Fuchs, A.R. Lehmann, C.M. Green, UbiquitinPCNA fusion as a mimic for mono-ubiquitinated PCNA in Schizosaccharomyces pombe, DNA Repair (Amst.) 9 (2010) 777-784.

[28] Z. Qin, M. Lu, X. Xu, M. Hanna, N. Shiomi, W. Xiao, DNA-damage tolerance mediated by PCNA*Ub fusions in human cells is dependent on Rev1 but not Poleta,
Nucleic Acids Res, 41 (2013) 7356-7369.

[29] H. Arakawa, D. Lodygin, J.M. Buerstedde, Mutant loxP vectors for selectable marker recycle and conditional knock-outs, BMC Biotechnol. 1 (2001) 7.

[30] A.L. Ross, L.J. Simpson, J.E. Sale, Vertebrate DNA damage tolerance requires the Cterminus but not BRCT or transferase domains of REV1, Nucleic Acids Res. 33 (2005) 1280-1289.

[31] D.J. Fitzgerald, P. Berger, C. Schaffitzel, K. Yamada, T.J. Richmond, I. Berger, Protein complex expression by using multigene baculoviral vectors, Nat. Methods 3 (2006) 1021-1032.

[32] J. Molnár, A. Póti, O. Pipek, M. Krzystanek, N. Kanu, C. Swanton, G.E. Tusnády, Z. Szállási, I. Csabai, D. Szüts, The genome of the chicken DT40 bursal lymphoma cell line, G3 (Bethesda) 4 (2014) 2231-2240.

[33] H. Arakawa, G.L. Moldovan, H. Saribasak, N.N. Saribasak, S. Jentsch, J.M. Buerstedde, A role for PCNA ubiquitination in immunoglobulin hypermutation, PLoS Biol. 4 (2006) e366.

[34] C.J. Vandenberg, F. Gergely, C.Y. Ong, P. Pace, D.L. Mallery, K. Hiom, K.J. Patel, BRCA1-independent ubiquitination of FANCD2, Mol. Cell 12 (2003) 247-254.

[35] Y. Yonetani, H. Hochegger, E. Sonoda, S. Shinya, H. Yoshikawa, S. Takeda, M. Yamazoe, Differential and collaborative actions of Rad51 paralog proteins in cellular response to DNA damage, Nucleic Acids Res. 33 (2005) 4544-4552.

[36] A. Varga, A.P. Marcus, M. Himoto, S. Iwai, D. Szuts, Analysis of CPD ultraviolet lesion bypass in chicken DT40Cells: polymerase eta and PCNA ubiquitylation play identical roles, PLoS One 7 (2012) e52472.

[37] J. Zamborszky, B. Szikriszt, J.Z. Gervai, O. Pipek, A. Poti, M. Krzystanek, D. Ribli, J.M. Szalai-Gindl, I. Csabai, Z. Szallasi, et al., Loss of BRCA1 or BRCA2 markedly increases the rate of base substitution mutagenesis and has distinct effects on genomic deletions, Oncogene 36 (2017) 746-755.

[38] O. Pipek, D. Ribli, J. Molnar, A. Poti, M. Krzystanek, A. Bodor, G.E. Tusnady, Z. Szallasi, I. Csabai, D. Szuts, Fast and accurate mutation detection in whole genome sequences of multiple isogenic samples with IsoMut, BMC Bioinf. 18 (2017) 73.

[39] Y. Masuda, M. Suzuki, H. Kawai, A. Hishiki, H. Hashimoto, C. Masutani, T. Hishida, F. Suzuki, K. Kamiya, En bloc transfer of polyubiquitin chains to PCNA in vitro is mediated by two different human E2-E3 pairs, Nucleic Acids Res. 40 (2012) 10394-10407.

[40] A. Hendel, P.H. Krijger, N. Diamant, Z. Goren, P. Langerak, J. Kim, T. Reissner, K.Y. Lee, N.E. Geacintov, T. Carell, et al., PCNA ubiquitination is important, but not essential for translesion DNA synthesis in mammalian cells, PLoS Genet. 7 (2011) e1002262.

[41] M.D. Wyatt, D.L. Pittman, Methylating agents and DNA repair responses: methylated bases and sources of strand breaks, Chem. Res. Toxicol. 19 (2006) 1580-1594.

[42] A. Eastman, Reevaluation of interaction of cis-dichloro(ethylenediamine)platinum (II) with DNA, Biochemistry 25 (1986) 3912-3915.

[43] S.F. Bunting, E. Callen, N. Wong, H.T. Chen, F. Polato, A. Gunn, A. Bothmer, N. Feldhahn, O. Fernandez-Capetillo, L. Cao, et al., 53BP1 inhibits homologous recombination in Brca1-deficient cells by blocking resection of DNA breaks, Cell 141 (2010) 243-254.

[44] Y. Qing, M. Yamazoe, K. Hirota, D. Dejsuphong, W. Sakai, K.N. Yamamoto, D.K. Bishop, X. Wu, S. Takeda, The epistatic relationship between BRCA2 and the other RAD51 mediators in homologous recombination, PLoS Genet. 7 (2011) e1002148.

[45] F. Zhang, J. Ma, J. Wu, L. Ye, H. Cai, B. Xia, X. Yu, PALB2 links BRCA1 and BRCA2 in the DNA-damage response, Curr. Biol. 19 (2009) 524-529.

[46] D.K. Bishop, U. Ear, A. Bhattacharyya, C. Calderone, M. Beckett, R.R. Weichselbaum, A. Shinohara, Xrcc3 is required for assembly of Rad51 complexes in vivo, J. Biol. Chem. 273 (1998) 21482-21488.

[47] H. Gali, S. Juhasz, M. Morocz, I. Hajdu, K. Fatyol, V. Szukacsov, P. Burkovics, L. Haracska, Role of SUMO modification of human PCNA at stalled replication fork, Nucleic Acids Res. 40 (2012) 6049-6059.

[48] B. Pfander, G.L. Moldovan, M. Sacher, C. Hoege, S. Jentsch, SUMO-modified PCNA recruits Srs2 to prevent recombination during S phase, Nature (2005).

[49] N.S. Tomi, K. Davari, D. Grotzky, F. Loos, K. Bottcher, S. Frankenberger, B. Jungnickel, Analysis of SHPRH functions in DNA repair and immunoglobulin diversification, DNA Repair (Amst.) 24 (2014) 63-72.

[50] A. Ciccia, A.V. Nimonkar, Y. Hu, I. Hajdu, Y.J. Achar, L. Izhar, S.A. Petit, B. Adamson, J.C. Yoon, S.C. Kowalczykowski, et al., Polyubiquitinated PCNA recruits the ZRANB3 translocase to maintain genomic integrity after replication stress, Mol. Cell 47 (2012) 396-409.

[51] K.J. Neelsen, M. Lopes, Replication fork reversal in eukaryotes: from dead end to dynamic response, Nat. Rev. Mol. Cell Biol. 16 (2015) 207-220.

[52] K. Schlacher, N. Christ, N. Siaud, A. Egashira, H. Wu, M. Jasin, Double-strand break repair-independent role for BRCA2 in blocking stalled replication fork degradation by MRE11, Cell 145 (2011) 529-542.

[53] A. Motegi, R. Sood, H. Moinova, S.D. Markowitz, P.P. Liu, K. Myung, Human SHPRH suppresses genomic instability through proliferating cell nuclear antigen polyubiquitination, J. Cell Biol. 175 (2006) 703-708. 